\title{
Spectrum Sensing Performance in Cognitive Radio Networks With Multiple Primary Users
}

\author{
António Furtado, Luís Irio, Rodolfo Oliveira, Member, IEEE, Luís Bernardo, Member, IEEE, and \\ Rui Dinis, Senior Member, IEEE
}

\begin{abstract}
Radio spectrum sensing (SS) has been an active topic of research over the past years due to its importance to cognitive radio (CR) systems. However, in CR networks (CRNs) with multiple primary users (PUs), the secondary users (SUs) can often detect PUs that are located outside the sensing range, due to the level of the aggregated interference caused by the PUs. This effect, known as spatial false alarm (SFA), degrades the performance of CRNs because it decreases the SUs' medium access probability. This paper characterizes the SFA effect in a CRN, identifying possible actions to attenuate it. Adopting energy-based sensing (EBS) in each SU, this paper starts to characterize the interference caused by multiple PUs located outside a desired sensing region. The interference formulation is then used to write the probabilities of detection and false alarm, and closed-form expressions are presented and validated through simulation. The first remark to be made is that the SFA can be neglected, depending on the path-loss factor and the number of samples collected by the energy detector to decide the spectrum's occupancy state. However, it is shown that by increasing the number of samples needed to increase the sensing accuracy, the SUs may degrade their throughput, namely, if SUs are equipped with a single radio that is sequentially used for sensing and transmission (split-phase operation). Assuming this scenario, this paper ends by providing a bound for the maximum throughput achieved in a CRN with multiple active PUs and for a given level of PUs' detection inside the SUs' sensing region. The results presented in this paper show the impact of path loss and EBS parameterization on SUs' throughput and are particularly useful to guide the design and parameterization of multihop CRNs, including future ad hoc CRNs considering multiple PUs.
\end{abstract}

Index Terms-Aggregate interference, cognitive radio (CR), energy detector.

Manuscript received July 18, 2014; revised November 13, 2014; accepted January 24, 2015. Date of publication February 20, 2015; date of current version March 10, 2016. This work was supported by the Foundation for Science and Technology of the Portuguese Ministry of Education and Science under Project MANY2COMWIN (EXPL/EEI-TEL/0969/2013), Project ADIN (PTDC/EEITEL/2990/2012), Project COPWIN (PTDC/EEI-TEL/1417/2012), and Project IT (UID/EEA/50008/2013), and under Fellowship SFRH/BD/88140/2012. The review of this paper was coordinated by Dr. J.-C. Chen.

A. Furtado, R. Oliveira, L. Bernardo, and R. Dinis are with the Centre of Technology and Systems, Instituto de Desenvolvimento de Novas Tecnologias, Departamento de Engenharia Electrotécnica, Faculdade de Ciências e Tecnologia, Universidade Nova de Lisboa, 2829-516 Lisbon, Portugal, and are also with Instituto de Telecomunicações (IT), 1049-001 Lisboa, Portugal (e-mail: a.furtado@campus.fct.unl.pt; rado@fct.unl.pt; 1flb@fct.unl.pt; rdinis@fct. unl.pt).

L. Irio is with the Centre of Technology and Systems, Instituto de Desenvolvimento de Novas Tecnologias, Departamento de Engenharia Electrotécnica, Faculdade de Ciências e Tecnologia, Universidade Nova de Lisboa, 2829-516 Lisbon, Portugal (e-mail: 1.irio@campus.fct.unl.pt).

Color versions of one or more of the figures in this paper are available online at http://ieeexplore.ieee.org.

Digital Object Identifier 10.1109/TVT.2015.2406254

\section{INTRODUCTION}

C OGNITIVE RADIO (CR) has been proposed as a solution to alleviate the increasing demand for radio spectrum [1]. The nodes equipped with CRs, which are usually denominated secondary users (SUs), must be aware of the activity of the licensed users, which are denominated primary users (PUs), to dynamically access the spectrum without causing them harmful interference.

SUs ensure a level of protection to PUs by using spectrum sensing (SS) techniques. SS plays a central role in CRNs. The sensing aims at detecting the availability of vacant portions (holes) of spectrum and has been a topic of considerable research over the last years [2]. The traditional SS techniques include waveform-based sensing (WBS) [3], which is a coherent technique that consists of correlating the received signal with a priori known set of different waveform patterns; matchedfilter-based sensing (MFBS) [4], which is an optimal sensing scheme where the received signal is also correlated with a copy of the transmitted one; and cyclostationarity-based sensing (CBS) [5], which is a technique that exploits the periodic characteristics of the received signals, i.e., carrier tones, pilot sequences, etc. Additionally, several sensing techniques have been recently proposed and briefly summarized in [6] and [7].

MFBS assumes prior knowledge of the primary's signal, whereas WBS assumes that the received signal matches with one of the patterns previously known. This means that these sensing techniques are not feasible in some bands, where several communication technologies may operate without a priori knowledge. On other hand, CBS is impracticable for signals that do not exhibit cyclostationarity properties.

Energy-based sensing (EBS) is the simplest SS technique, and its main advantage is related to the fact that it needs no a priori knowledge of PU's signal. This paper considers that SUs adopt EBS. It is well known that EBS can exhibit low performance in specific comparative scenarios [8] or when noise's variance is unknown or very large. EBS has been studied in several CR scenarios, namely on local and cooperative sensing schemes [2]. More recently, several EBS schemes adopting subNyquist sampling have been proposed, which are advantageous in terms of the sensing duration [7]. The energy-based detection principle employed in EBS was first studied by Urkowitz, who formulated the problem as a binary hypothesis testing for the detection of deterministic signals considering white [9] and colored [10] Gaussian noise. The analysis of energybased detection was extended by Kostylev [11] to signals with random amplitude caused by fading effects. Similar analysis of 
energy-based detection was also considered in [12], which provides a closed-form expression for the probability of detection for Rayleigh, Nakagami, and Rician fading channels. Similar assumptions were formally treated in a different way in [13] and [14]. In [15], an analysis of energy detection performance considering generalized $\kappa-\mu$ extreme fading channels is presented. The previous studies have considered fast-fading channels. In [16], an approximation used to compute the probability of detection in slow fading channels was derived.

In the aforementioned papers, a single PU is considered. The methodology to write the probabilities of detection and false alarm is based on the assumption that the distribution of both hypotheses is Gaussian. While relying on the central limit theorem (CLT), the number of samples required to observe this assumption is generally high, and its impact on the SUs' throughput cannot be negligible. The detection and false alarm probabilities are used to define the decision threshold. The majority of works simply compute the decision threshold for a required probability of detection or false alarm [14], which is known as the constant false-alarm ratio criterion. However, another parameterization criteria can be found in the literature: In [17], a decision threshold parameterization imposing the probabilities of detection and false alarm was proposed, which maximizes the SUs' medium access probability for a given probability of channel availability. More parameterization criteria were presented and compared in [18].

Independently of the criteria rationale, the SUs parameterize the SS technique to ensure that the PUs located in a certain sensing region have a given level of protection. SU's sensing range is usually parameterized to detect the farthest PU that would not tolerate the interference caused when the SU transmits. By doing so, an SU can detect the farthest PU to which the SU may interfere with, and the $\mathrm{SU}$ is only granted to access the channel when no PU is detected. However, there are several scenarios where one or more PUs located outside the sensing region can be detected by an SU. In this case, an SU cancels its transmission, reducing its performance. This effect of an SU misinterpreting a noninterfering PU was first studied in [19], where it was called the spatial false alarm (SFA) problem.

In SFA, the characterization of the interference caused by the PUs outside the sensing region is of particular importance. In [20]-[24], several analytical approaches to model the aggregate interference in static networks were presented. However, due to the considered assumptions, the aggregate distribution of the interference cannot be approximated by a Gaussian distribution, which increases the complexity of the sensing performance analytical characterization.

In [19], the SFA problem was introduced, showing that it is caused by the deviation of test statistics of the received PU signal and occurs for various sensing techniques. By characterizing the probability of detection of a single PU when it is located inside and outside a defined SU's sensing region, it has been concluded that it is inevitable that a PU could be sensed by an SU, even when located outside the sensing region. The impact of neglecting the SFA problem in the throughput of an SU is also characterized. The work in [19] has been recently extended in [25], where the SU's probability of accessing the channel considering the existence of multiple PUs was charac- terized. From the theoretical analysis, general upper and lower bounds of the SU's sensing performance are derived. In [19], it shown that the constant-false-alarm-rate criterion, which is usually employed to parameterize the SS, should act on the SFA probability instead of the conventional false-alarm probability. This is due to the fact that multiple PUs may be active outside the sensing region.

Different from Weijia's work in [25], which have characterized the probability of medium access achieved by the SUs when multiple PUs cause the SFA effect, this paper proposes a different formal treatment to characterize different performance metrics. The main contributions of this paper can be summarized as follows.

- This paper characterizes the performance of the cognitive network in terms of the conditional throughput achieved by the SUs. In this way, the impact of the number of samples needed to reach the required PU's level of protection is taken into account. This is an important point because the number of samples needed to impose the required level of detection may be too high under certain circumstances. Moreover, and even more important, the throughput also accounts with the case when a node accesses the medium without success due to a false alarm, being less optimistic than the probability of medium access characterized in [25].

- Since the path-loss coefficient deeply impacts on the SFA effect, we present several results showing the impact of the path loss on the probabilities of detection and false alarm and on the conditional throughput achieved by the SUs. As shown in the last figure in this paper, as the pathloss coefficient increases, the throughput achieved by the SUs decrease. Moreover, important is the fact that the path-loss coefficient may restrict the level of interference protection to PUs to values where it is forbidden to operate due to the nontolerable probability of miss detection.

- Departing from the observation that the number of samples required by the energy detector to reach the sensing requirements is usually high, we have observed that fading channels improve the detection probability. In line with this observation, we simplify the methodology of energy-detector decision threshold parameterization by not considering the fading effects in the amplitude of the received signals. In this way, only path loss is considered, which frequently represents the worst-case scenario in terms of the probability of detection.

Apart from the given contributions, our approach introduces several contributions related with the methodology proposed to characterize the probabilities of detection and false alarm, as well as the throughput achieved by the SUs.

- A Gaussian approximation is first derived for the distribution of the aggregate interference caused by the PUs located in a circular ring, and its accuracy is assessed through simulation.

- Considering that the PUs are spatially distributed according to a 2-D Poisson point process and are active with probability $\rho_{\mathrm{ON}}$, we derive the SU's probability of 
detecting and erroneously detecting (false alarm) PU's activity in the sensing region. These probabilities are then used to formulate a solution to parameterize the energy detector decision threshold.

- Using the distribution of the aggregate interference generated within and outside the sensing region to parameterize the decision threshold, we propose an optimization problem to find the minimum number of samples required to meet the PUs' protection level, which simultaneously maximize the throughput achieved by the SUs. While similar optimization problems aiming to maximize the SUs' throughput subject to detection constraints have been proposed [26], our contribution is essentially related with the statement of such an optimization for the SFA scenario.

- Different results are presented for the probabilities of PUs' detection and false alarm in different propagation scenarios and considering different numbers of channel samples. The results presented in this paper show that depending on the path-loss coefficient and on the number of samples to support the channel's occupancy decision, the SFA effect may be attenuated and/or almost neglected.

- Finally, the upper bound for the throughput achieved by a CRN where each SU is equipped with a single radio is provided and characterized for several propagation conditions and EBS parameterizations. The bound captures the impact of the SFA for different CRN conditions.

Section II introduces the considered system. Section III describes the steps involved to model the aggregate power received by an SU from PUs located in a circular ring, presenting simulation results that successfully validate the proposed solution. Section IV derives the probabilities of detection and false alarm when EBS is considered, which are also validated through simulation. Finally, Section V characterizes the SS performance when multiple PUs are considered and evaluates its impact on CRN's throughput. Final remarks are given in Section VI.

\section{SYSTEM DESCRIPTION}

This paper considers a cognitive radio network (CRN) such as the one shown in Fig. 1. We consider that each $\mathrm{SU}$ has a given range of sensing, which is limited by the radius $R_{G}$. Within the sensing region $\mathcal{A}_{g}$ with area $A_{G}=\pi R_{G}^{2}$ (white zone in the figure), an active PU must be detected with a given probability $P_{D}$ to guarantee a certain level of protection to PUs due to SUs' transmissions. Note that $R_{G}$ should be chosen in such a way to ensure that a PU outside the sensing region tolerates an SU transmission located in the center of the sensing region. PUs are also located outside the sensing region (gray zone), causing interference to the SU sensing the spectrum.

The primary users are distributed within a certain area $A_{E}=$ $\pi R_{E}^{2}$ encircling the SU transmitter. The number of PUs is represented by a random variable $X$. In this paper, it is considered that the PUs are distributed according to a 2-D Poisson point process, with the following distribution:

$$
\mathrm{P}_{A}(X=l)=\frac{\left(\rho_{\mathrm{ON}} \beta A\right)^{l}}{l !} e^{-\rho_{\mathrm{ON}} \beta A}, \quad l=0,1, \ldots N
$$

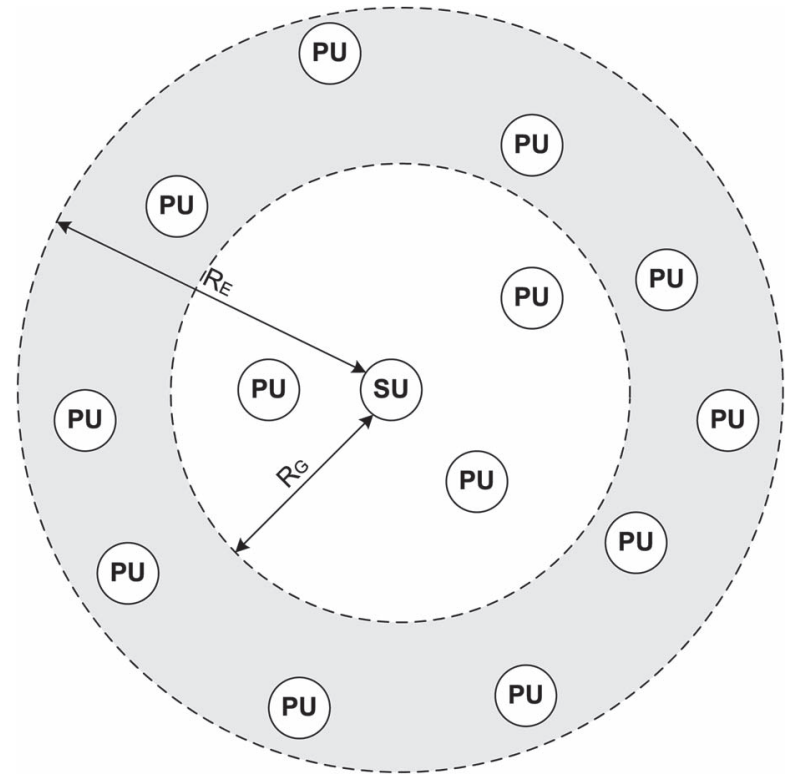

Fig. 1. Sensing (white) and interference (gray) regions.

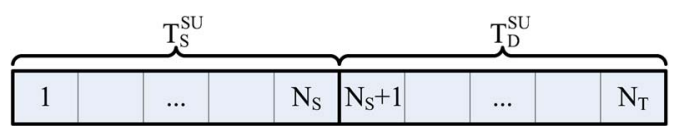

Fig. 2. SU's time frame structure.

where $\beta$ is the PU's spatial density, $A$ is the total area where the PUs are distributed, $\rho_{\mathrm{ON}}$ represents the probability of finding a PU active, and $N$ is the maximum number of PUs.

Single-radio SUs are considered, meaning that SUs are equipped with a single transceiver; therefore, the SUs are unable to sense and transmit simultaneously. Due to this limitation, SUs adopt an operation cycle where sensing and transmission operations occur in a consecutive manner. SUs start to sense the spectrum during a fixed amount of time (sensing period), and depending on the output of the sensing, they can transmit in the sensed band during a fixed amount of time (transmission period). SUs repeat the operation cycle periodically to minimize the amount of interference caused to licensed users. In this way, each SU may access the channel opportunistically when one or more PUs do not use the channel, as we have considered in [27]. SUs adopt an EBS technique. The duration of SU's sensing and transmitting periods is denoted $T_{S}^{\mathrm{SU}}$ and $T_{D}^{\mathrm{SU}}$, respectively, as shown in Fig. 2.

The time frame of the SUs is divided into $N_{T}$ slots where each slot duration is given by the channel sampling period adopted by the energy detector. The first $N_{S}$ slots are allocated to the SS task (for channel sampling), and the remaining ones $\left(N_{S}+1\right.$ to $\left.N_{T}\right)$ are used to access the channel (for transmission, whenever possible).

Finally, the notation adopted in this paper is described in Table I.

\section{Aggregate Interference}

As described in [19] and [25], the SFA problem is due to the interference caused by the PUs located outside the sensing 